\title{
The fate of non-trivial entanglement under gravitational collapse
}

\author{
Eduardo Martín-Martínez \\ Institute for Quantum Computing, Department of Physics and Astronomy, and Department of Applied Mathematics, \\ University of Waterloo, 200 University Avenue W, Waterloo, Ontario, N2L 3G1, Canada
}

\section{Luis J. Garay}

Departamento de Física Teórica II, Universidad Complutense de Madrid, 28040 Madrid, Spain

Instituto de Estructura de la Materia, CSIC, Serrano 121, 28006 Madrid, Spain

\author{
Juan León \\ Instituto de Física Fundamental, CSIC, Serrano 113-B, 28006 Madrid, Spain
}

\begin{abstract}
We analyse the evolution of the entanglement of a non-trivial initial quantum field state (which, for simplicity, has been taken to be a bipartite state made out of vacuum and the first excited state) when it undergoes a gravitational collapse. We carry out this analysis by generalising the tools developed to study entanglement behaviour in stationary scenarios and making them suitable to deal with dynamical spacetimes. We also discuss what kind of problems can be tackled using the formalism spelled out here as well as single out future avenues of research.
\end{abstract}




\section{Introduction}

The question of how entanglement behaves in non-inertial frames and in curved spacetimes has been around for already a fairly long time. There are many works that centre in the study of uniformly accelerated observers (among many others [1, 2, 3, 4, 5, 6, 7, 8, 9, 10]), or in the background of a stationary eternal black hole [11. There are also some studies involving entanglement dynamics in expanding universe scenarios which have shown that the interaction with the gravitational field can produce entanglement between quantum field modes [12, 13].

Focusing on the problem of gravitational collapse, previous works in the literature analysed the correlations between the outgoing and infalling modes in a gravitational collapse when the initial state is the vacuum (see for example [14, 15, 16, 17, 13, again among many others).

In this work we consider the following more involved but central issue as far as the behaviour of entanglement in a dynamical spacetime is concerned. In the asymptotic past the field lives in a flat spacetime and its state has some degree of quantum entanglement between two of its modes. Then at some point, gravitational collapse occurs. The collapse makes the observers of the field unable to access the full state due to the formation of an event horizon. This has an impact on the entanglement that any observer of the field state can acknowledge.

Studying this sort of problems is interesting from many perspectives apart from understanding how quantum correlations behave in dynamical curved spacetimes. Quantum entanglement plays a key role in black hole thermodynamics and the fate of information in the presence of horizons. Also, the study of the behaviour of nontrivial quantum entanglement in gravitational collapse may be useful for analog gravity proposals that aim at making use of this entanglement as a resource to check genuinely quantum effects derived form the formation of a horizon [17. In general, this will arguably constitute a rather difficult exercise. However, inspired by tools developed to study the effect of accelerations on quantum entanglement, it may be possible to shed some light on this problem.

In the study of quantum entanglement from non-inertial perspectives, i.e. in the context of relativistic quantum information, it was not until relatively recently that the physical meaning of the so-called 'single mode approximation' was analysed in detail. This approximation was introduced in 1, 18. It consisted in assuming that the Bogoliubov transformations between Minkowski and Rindler modes did not mix frequencies. In 2010 appropriate procedures to construct inertial modes which transform to monochromatic Rindler modes were introduced in the context of Relativistic Quantum Information for the accelerated scenario [7] as well as in the stationary Schwarzschild scenario [11. Nowadays we are taking steps towards the analysis of localised states 19 and entanglement behaviour in modes contained in cavities [9, 10]. Although there are still a number of open questions about the analysis of localised field states and their possible experimental implementability, the two milestones [1, 18, and [7 have enabled us to understand better the way in which entanglement behaves from non-inertial perspectives. And so, we analyse here the fundamental and qualitative effect of a dynamical gravitational collapse on bipartite entanglement contained in non-trivial quantum field states (that involve vacuum and excited states) prior to the collapse.

In Sec. 2 we introduce the basic spacetime and quantum-field ingredients and tools to analyse the fate of entanglement in a gravitational collapse scenario. Section 3 is devoted to the study of the evolution of the entanglement of a specific bipartite quantum field state made out of vacuum and an excited state (to our knowledge, this is the first time that this kind of non-trivial entanglement in a dynamical spacetime is analysed). Section 4 contains the conclusion and some lines of future research in this context.

\section{Gravitational Collapse}

We will consider a certain maximally entangled state of two modes of the field. This entangled state lives in a spacetime that is originally flat. At some point, a perturbation is produced causing the spacetime to undergo a process of gravitational collapse. This scenario would very well describe the process of an astrophysical stellar collapse: Prior to the collapse the density of a star is small enough to consider that the spacetime is approximately flat. At some point, the internal forces that kept the star from collapsing fail to counter the gravitational interaction and the star collapses. If nothing stops the collapse, it will reach a point in which an event horizon is formed.

Let us consider the following metric written in terms of ingoing Eddington-Finkelstein coordinates as

$$
\mathrm{d} s^{2}=-\left(1-\frac{2 M(v)}{r}\right) \mathrm{d} v^{2}+2 \mathrm{~d} v \mathrm{~d} r+r^{2} \mathrm{~d} \Omega^{2},
$$

where $r$ is the radial coordinate, $v$ is the ingoing null coordinate, and $M(v)=m \theta\left(v-v_{0}\right)$. For $v_{0}<v$ this is nothing but the ingoing Eddington-Finkelstein representation for the Schwarzschild metric whereas for $v<v_{0}$ it is just Minkowski spacetime. This metric represents a radial ingoing collapsing shockwave of radiation and it is called Vaidya metric (described schematically in Fig. 11). This metric is a solution to the Einstein equations (see for instance. Ref. [15]) that, in spite of its simplicity, describes very well the gravitational collapse scenario that we want to analyse. Refinements of the model to make it more realistic only introduce subleading corrections. In particular this model captures, up to 


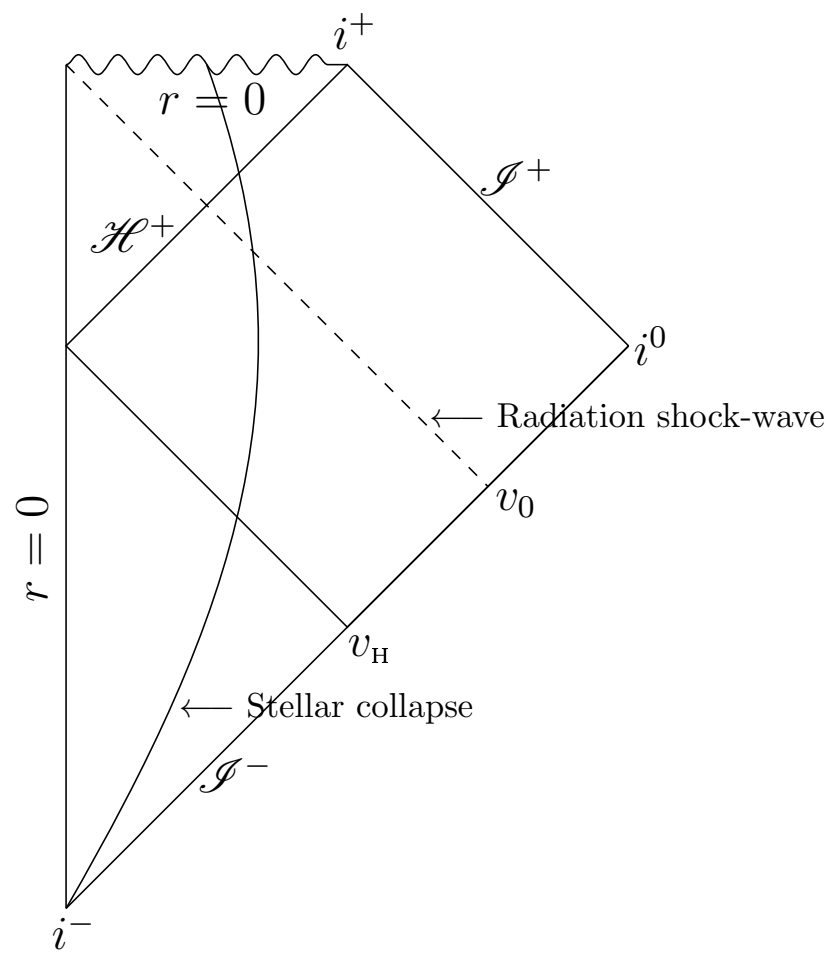

Figure 1. Carter-Penrose diagrams for gravitational collapse: Stellar collapse (solid line) and ingoing radiation shockwave (dashed line) giving rise to Vaidya spacetime.

subleading corrections, the more realistic collapse of a matter cloud. Let $v_{\mathrm{H}}=v_{0}-4 m$ be the coordinate of the last null ray that escapes to the future null infinity $\mathscr{I}^{+}$and hence that will eventually form the event horizon (see Fig. 1).

Consider now a state of a scalar quantum field. We need to introduce convenient bases of solutions to the KleinGordon equation determined by their behaviour in the different relevant regions of this collapsing spacetime. For this, we will follow a standard procedure (see e.g. [15]).

We first define the 'in' basis of ingoing positive frequency modes, associated with the time parameter $v$ at the null past infinity $\mathscr{I}^{-}$:

$$
u_{\omega}^{\text {in }} \sim(4 \pi r \sqrt{\omega})^{-1} e^{-\mathrm{i} \omega v}, \quad \text { at } \mathscr{I}^{-} .
$$

Second, we define another basis in a Cauchy surface in the future. In this case, the asymptotic future $\mathscr{I}^{+}$is not a Cauchy surface in itself, so we need to consider also the future event horizon $\mathscr{H}^{+}$. Let us begin with the 'out' modes defined as being outgoing positive-frequency in terms of the natural time parameter $\eta_{\text {out }}$ at $\mathscr{I}^{+}$, which are

$$
u_{\omega}^{\text {out }} \sim(4 \pi r \sqrt{\omega})^{-1} e^{-\mathrm{i} \omega \eta_{\text {out }}}, \quad \text { at } \mathscr{I}^{+},
$$

where $\eta_{\text {out }}=v-2 r_{\text {out }}^{*}$ and $r_{\text {out }}^{*}$ is the radial tortoise coordinate in the Schwarzschild region. At early times, these modes $u_{\omega}^{\text {out }}$ concentrate near $v_{\mathrm{H}}$ at $\mathscr{I}^{-}$and behave in the following way:

$$
u_{\omega}^{\text {out }} \approx(4 \pi r \sqrt{\omega})^{-1} e^{-\mathrm{i} \omega\left(v_{\mathrm{H}}-4 m \ln \frac{\left|v_{\mathrm{H}}-v\right|}{4 m}\right)} \theta\left(v_{\mathrm{H}}-v\right), \quad \text { at } \mathscr{I}^{-},
$$

having support only in the region $v<v_{\mathrm{H}}$, since only the rays of light that depart from $v<v_{\mathrm{H}}$ will reach the asymptotic region $\mathscr{I}^{+}$. The rest will fall down into the horizon.

Finally, we use an analytical continuation argument to define the 'hor' modes at $\mathscr{H}^{+}$: These will be modes that behave as $u_{\omega}^{\text {out }}$ in the asymptotic past $\mathscr{I}^{-}$, but for $v>v_{\mathrm{H}}$. In other words, we define them as modes that leave the asymptotic past to fall into the horizon, never reaching the asymptotic future. Near the Cauchy surface $\mathscr{I}^{-}$, these modes behave as

$$
u_{\omega}^{\text {hor }} \sim(4 \pi r \sqrt{\omega})^{-1} e^{+\mathrm{i} \omega\left(v_{\mathrm{H}}-4 m \ln \frac{\left|v_{\mathrm{H}}-v\right|}{4 m}\right)} \theta\left(v-v_{\mathrm{H}}\right), \quad \text { at } \mathscr{I}^{-} .
$$

By expanding the field in terms of the two sets of modes ('in' on the one hand and 'hor-out' on the other) we can relate the two sets of solutions via the corresponding Bogoliubov coefficients. We will provide more details below, but 
for now let us refer to the extensive literature on this topic (see for instance [15]) and directly give the expression of the bosonic annihilation field operators in the asymptotic past in terms of the creation and annihilation operators of 'out' and 'hor' modes which, in the notation of [13], read:

$$
a_{\omega^{\prime}}^{\text {in }}=\int d \omega\left[\alpha_{\omega \omega^{\prime}}^{*}\left(a_{\omega}^{\text {out }}-\tanh r_{\omega} a_{\omega}^{\text {hor }^{\dagger}}\right)+\alpha_{\omega \omega^{\prime}} e^{i \varphi}\left(a_{\omega}^{\text {hor }}-\tanh r_{\omega} a_{\omega}^{\text {out }}{ }^{\dagger}\right)\right],
$$

where $\tanh r_{\omega}=e^{-4 \pi m \omega}$. The values of $\varphi$ and $\alpha_{\omega \omega^{\prime}}$ will be given below. The 'in' vacuum, given by $a_{\omega}^{\text {in }}|0\rangle_{\text {in }}=0$ for all positive frequencies $\omega$, can be readily rewritten in the 'out-hor' basis as

$$
|0\rangle_{\text {in }}=\prod_{\omega} \frac{1}{\cosh r_{\omega}} \sum_{n=0}^{\infty}\left(\tanh r_{\omega}\right)^{n}\left|n_{\omega}\right\rangle_{\text {hor }}\left|n_{\omega}\right\rangle_{\text {out }}
$$

where $\left|n_{\omega}\right\rangle$ denotes a mode with occupation number $n$ and frequency $\omega$.

The next task is to write the one-particle state in the past $\left|1_{\omega}\right\rangle_{\text {in }}$ as a linear combination of the 'out-hor' basis modes. If we have a monochromatic excitation in the asymptotic past, equation (6) tells us that it will become a highly non-monochromatic linear combination of 'hor' and 'out' modes. The standard well-known procedure (which we briefly summarise in what follows, see e.g. [15, 7]) is to construct another basis of 'in' modes with positive-frequency in the past such that its Bogoliubov transformation into 'hor' and 'out' modes is diagonal in frequencies. Let us call those modes $u_{\Omega}^{\mathrm{R}}$ and $u_{\Omega}^{\mathrm{L}}$ in order to keep the notation of Ref. [7. Note that, for all the reasons discussed above, these modes are intrinsically non-monochromatic in the asymptotic past, i.e. in the basis of modes $u_{\omega}^{\text {in }}$, and that $\Omega$ is labelling the frequency of such modes $u_{\Omega}^{\mathrm{R}}$ and $u_{\Omega}^{\mathrm{L}}$ with respect to the time in the asymptotic future region 'out'.

As suggested by the fact that $|0\rangle_{\text {in }}$ is a two-mode squeezed vacuum of 'out' and 'hor' modes, let us define new positive-norm 'R-L' modes by the following diagonal Bogoliubov transformation from 'out-hor' basis with the form of a two-mode squeezing operation:

$$
\begin{aligned}
& u_{\Omega}^{\mathrm{R}}=\cosh \left(r_{\Omega}\right) u_{\Omega}^{\text {out }}+\sinh \left(r_{\Omega}\right) u_{\Omega}^{\text {hor* }}, \\
& u_{\Omega}^{\mathrm{L}}=\cosh \left(r_{\Omega}\right) u_{\Omega}^{\text {hor }}+\sinh \left(r_{\Omega}\right) u_{\Omega}^{\text {out* }} .
\end{aligned}
$$

Taking Klein-Gordon inner products with the 'in' modes we find the following form of $u_{\Omega}^{\mathrm{R}}$ and $u_{\Omega}^{\mathrm{L}}$ in terms of the $u_{\omega}^{\text {in }}$ modes:

$$
\begin{aligned}
& u_{\Omega}^{\mathrm{R}}=\int_{0}^{\infty}\left[\cosh \left(r_{\Omega}\right) \alpha_{\omega \Omega}+\sinh \left(r_{\Omega}\right) \delta_{\omega \Omega}\right]^{*} u_{\omega}^{\mathrm{in}} \mathrm{d} \omega, \\
& u_{\Omega}^{\mathrm{L}}=\int_{0}^{\infty}\left[\cosh \left(r_{\Omega}\right) \gamma_{\omega \Omega}+\sinh \left(r_{\Omega}\right) \beta_{\omega \Omega}\right]^{*} u_{\omega}^{\mathrm{in}} \mathrm{d} \omega,
\end{aligned}
$$

where $\alpha_{\omega \Omega}=\left(u_{\omega}^{\text {in }}, u_{\Omega}^{\text {out }}\right), \beta_{\omega \Omega}=\left(u_{\omega}^{\text {in }}, u_{\Omega}^{\text {out }}\right), \gamma_{\omega \Omega}=\left(u_{\omega}^{\text {in }}, u_{\Omega}^{\text {hor }}\right)$ and $\delta_{\omega \Omega}=\left(u_{\omega}^{\text {in }}, u_{\Omega}^{\text {hor* }}\right)$ are the following Bogoliubov coefficients

$$
\begin{aligned}
& \alpha_{\omega \Omega}=\frac{-1}{2 \pi} \sqrt{\frac{\Omega}{\omega}}(4 m)^{-4 \mathrm{i} m \omega} e^{-\mathrm{i}(\omega-\Omega) v_{\mathrm{H}}}(-\mathrm{i} \Omega)^{-1-4 \mathrm{i} m \omega} \Gamma(1+4 \mathrm{i} m \omega) \\
& \beta_{\omega \Omega}=-\tanh r_{\omega} e^{-2 \mathrm{i} \Omega v_{\mathrm{H}}} \alpha_{\omega \Omega} \quad \gamma_{\omega \Omega}=e^{-2 \mathrm{i} \Omega v_{\mathrm{H}}} \alpha_{\omega \Omega} \quad \delta_{\omega \Omega}=-\tanh r_{\omega} \alpha_{\omega \Omega}^{*} .
\end{aligned}
$$

Therefore we see that the 'R-L' modes are purely positive frequency linear combinations of the 'in' modes and that they also form a complete set of solutions of the field equation in the asymptotic past.

This relationship between the modes directly translates into a relation between the particle operators associated with them

$$
\begin{aligned}
& a_{\Omega}^{\mathrm{R}}=\cosh r_{\Omega} a_{\Omega}^{\text {out }}-\sinh r_{\Omega} a_{\Omega}^{\text {hor }^{\dagger}}, \\
& a_{\Omega}^{\mathrm{L}}=\cosh r_{\Omega} a_{\Omega}^{\text {hor }}-\sinh r_{\Omega} a_{\Omega}^{\text {out }}{ }^{\dagger} .
\end{aligned}
$$

Obviously, these operators annihilate the 'in' vacuum: $a_{\Omega}^{\mathrm{R}}|0\rangle_{\text {in }}=a_{\Omega}^{\mathrm{L}}|0\rangle_{\text {in }}=0$.

To summarise, these modes have the following features:

(i) They share the same vacuum $|0\rangle_{\text {in }}$ as the monochromatic modes $a_{\omega}^{\text {in }}$.

(ii) They form a complete basis of solutions to the field equation which are positive-frequency in the asymptotic past.

(iii) They translate into a single frequency mode when expressed in the future basis. 
Properties (i) and (ii) allow us to decompose any physical state as some combination of these modes, which makes them worth studying as an intermediate stage of more general cases. The third feature (diagonal Bogoliubov transformations) greatly simplifies the formalism, enabling us to use all the artillery already deployed in other simpler scenarios also in the case of stellar collapse, providing us with a nice and clear interpretation to the analysis of the entanglement in the asymptotic future.

Repeating an analogous reasoning as in [7, we can still introduce a more general annihilation operator with this properties, which will be a linear combination of the two annihilation operators $a_{\Omega}^{\mathrm{R}}$ and $a_{\Omega}^{\mathrm{L}}$ defined above:

$$
C_{\Omega, q_{\mathrm{R}}}=q_{\mathrm{R}} a_{\Omega}^{\mathrm{R}}+q_{\mathrm{L}} a_{\Omega}^{\mathrm{L}},
$$

where $q_{\mathrm{L}}$ and $q_{\mathrm{R}}$ are real parameters satisfying $q_{\mathrm{L}}=\sqrt{1-q_{\mathrm{R}}^{2}}$ and $2^{-1 / 2} \leq q_{\mathrm{R}} \leq 1$.

\section{Entanglement behaviour}

Let us consider the following maximally entangled bipartite state in the asymptotic past, "prepared" long before collapse starts

$$
|\Psi\rangle=\frac{1}{\sqrt{2}}\left(|0\rangle_{\text {in }}^{\mathrm{A}}|0\rangle_{\text {in }}^{\mathrm{B}}+|1\rangle_{\text {in }}^{\mathrm{A}}\left|1_{\Omega}\right\rangle_{\text {in }}^{\mathrm{B}}\right),
$$

where the excited modes for Bob are chosen to be those generated by 15 , namely,

$$
\left|1_{\Omega}\right\rangle_{\text {in }}=C_{\Omega, q_{\mathrm{R}}}^{\dagger}|0\rangle_{\text {in }},
$$

whereas Alice's mode can be chosen arbitrarily (that is why it is not labelled with $\Omega$ ). This initial state (16) will be observed by two observers Alice and Bob. While we will consider that Alice has unrestricted access to her partial state, we will assume that Bob does not because at some point the process of gravitational collapse will generate an event horizon, preventing him from accessing the full state.

Although perhaps the most natural scenario would be that in which both subsystems are in the proximities of a stellar collapse and, hence, both observers would undergo similar processes, let us consider for simplicity that only one of the subsystems is going to be affected by the stellar collapse. One can think of Alice's state prepared such that it is a localized state living far away from the collapsing star. Alternatively we could consider that Alice can measure her subsystem prior to the formation of the horizon so that it cannot hinder her ability to obtain information about her partial state. On the other hand, Bob's knowledge about his subsystem is going to be limited because between the time when the state was created and the time in which he will be able to measure it, an event horizon appears, preventing him from accessing the full state in the future. This scenario is not devoid of physical interest. As we will show later on, it will allow us to focus on questions regarding quantum correlations between modes in the past and modes falling into the horizon.

In these circumstances, Alice measures in the 'in' basis whereas Bob measures in the 'out' basis, having lost all the information contained in the modes 'hor' that are bound to fall into the forming black hole. Now, to describe the effective state to which Alice and Bob can access requires that we trace out the modes that become causally disconnected from Bob due to the formation of the horizon, i.e. the 'hor' modes:

$$
\rho_{A-\text { out }}=\operatorname{tr}_{\text {hor }}(|\Psi\rangle\langle\Psi|) \text {. }
$$

This state is non-separable and we can compute its negativity [20] as a convenient quantifier of quantum entanglement. In simple words, when we compute the negativity of the density matrix $\rho_{A \text {-out }}$ we obtain a measurement of the correlations between Alice's fully accessible state and the modes that reach the asymptotic future escaping the collapse.

It is legit then to ask about what would be the quantum entanglement between Alice's state and those modes that will not make it to the asymptotic future because they will fall into the incipient horizon, becoming trapped into the black hole. The state whose separability we would have to analyse would then be

$$
\rho_{A-\text { hor }}=\operatorname{tr}_{\text {out }}(|\Psi\rangle\langle\Psi|) \text {. }
$$

The procedure to compute the negativity is the following: first we express $|\Psi\rangle$ in the basis of 'hor-out' modes for Bob, which yields

$$
\begin{aligned}
|\Psi\rangle & =\frac{1}{\sqrt{2}}\left[\frac{1}{\cosh r_{\Omega}} \sum_{n=0} \tanh ^{n} r_{\Omega}|0\rangle_{\text {in }}^{\mathrm{A}}\left|n_{\Omega}\right\rangle_{\text {out }}^{\mathrm{B}}\left|n_{\Omega}\right\rangle_{\text {hor }}^{\mathrm{B}}\right. \\
& \left.+\frac{1}{\cosh ^{2} r_{\Omega}} \sum_{n=0} \sqrt{n+1} \tanh ^{n} r_{\Omega}|1\rangle_{\text {in }}^{\mathrm{A}}\left(q_{\mathrm{R}}\left|(n+1)_{\Omega}\right\rangle_{\text {out }}^{\mathrm{B}}\left|n_{\Omega}\right\rangle_{\text {hor }}^{\mathrm{B}}+q_{\mathrm{L}}\left|n_{\Omega}\right\rangle_{\text {out }}^{\mathrm{B}}\left|(n+1)_{\Omega}\right\rangle_{\text {hor }}^{\mathrm{B}}\right)\right] .
\end{aligned}
$$


Then the density matrices $\rho_{A-\text { hor }}$ and $\rho_{A \text {-out }}$ are obtained after a simple but lengthy algebra exercise by tracing out the 'out' and 'hor' modes respectively from $|\Psi\rangle$,

$$
\begin{aligned}
& \rho_{A-\text { out }}=\sum_{n=0} \frac{T_{r}^{2 n}}{2 C_{r}^{2}}|0\rangle_{\text {in }}^{\mathrm{A}}\left|n_{\Omega}\right\rangle_{\text {out }}^{\mathrm{B}}\left\langle0 | _ { \text { in } } ^ { \mathrm { A } } \left\langle\left. n_{\Omega}\right|_{\text {out }} ^{\mathrm{B}}\right.\right. \\
& +\sum_{n=0}(n+1) \frac{T_{r}^{2 n}}{2 C_{r}^{4}}\left(q _ { \mathrm { R } } ^ { 2 } | 1 \rangle _ { \text { in } } ^ { \mathrm { A } } | ( n + 1 ) _ { \Omega } \rangle _ { \text { out } } ^ { \mathrm { B } } \langle 1 | _ { \text { in } } ^ { \mathrm { A } } \langle ( n + 1 ) _ { \Omega } | _ { \text { out } } ^ { \mathrm { B } } + q _ { \mathrm { L } } ^ { 2 } | 1 \rangle _ { \text { in } } ^ { \mathrm { A } } | n _ { \Omega } \rangle _ { \text { out } } ^ { \mathrm { B } } \left\langle\left.1\right|_{\text {in }} ^{\mathrm{A}}\left\langle\left. n_{\Omega}\right|_{\text {out }} ^{\mathrm{B}}\right)\right.\right. \\
& +\sum_{n=0} \sqrt{(n+1)(n+2)} \frac{T_{r}^{2 n+1}}{2 C_{r}^{4}} q_{\mathrm{R}} q_{\mathrm{L}}\left(| 1 \rangle _ { \text { in } } ^ { \mathrm { A } } | n _ { \Omega } \rangle _ { \text { out } } ^ { \mathrm { B } } \langle 1 | _ { \text { in } } ^ { \mathrm { A } } \langle ( n + 2 ) _ { \Omega } | _ { \text { out } } ^ { \mathrm { B } } + | 1 \rangle _ { \text { in } } ^ { \mathrm { A } } | ( n + 2 ) _ { \Omega } \rangle _ { \text { out } } ^ { \mathrm { B } } \left\langle\left.1\right|_{\text {in }} ^{\mathrm{A}}\left\langle\left. n_{\Omega}\right|_{\text {out }} ^{\mathrm{B}}\right)\right.\right. \\
& +\sum_{n=0} \sqrt{n+1} \frac{T_{r}^{2 n+1}}{2 C_{r}^{3}}\left[q _ { \mathrm { R } } \left(| 0 \rangle _ { \text { in } } ^ { \mathrm { A } } | n _ { \Omega } \rangle _ { \text { out } } ^ { \mathrm { B } } \langle 1 | _ { \text { in } } ^ { \mathrm { A } } \langle ( n + 1 ) _ { \Omega } | _ { \text { out } } ^ { \mathrm { B } } + | 1 \rangle _ { \text { in } } ^ { \mathrm { A } } | ( n + 1 ) _ { \Omega } \rangle _ { \text { out } } ^ { \mathrm { B } } \left\langle\left.0\right|_{\text {in }} ^{\mathrm{A}}\left\langle\left. n_{\Omega}\right|_{\text {out }} ^{\mathrm{B}}\right)\right.\right.\right. \\
& +q_{\mathrm{L}}\left(|0\rangle_{\text {in }}^{\mathrm{A}}\left|(n+1)_{\Omega}\right\rangle_{\text {out }}^{\mathrm{B}}\left\langle\left. 1\right|_{\text {in }} ^{\mathrm{A}}\left\langle\left. n_{\Omega}\right|_{\text {out }} ^{\mathrm{B}}+\mid 1\right\rangle_{\text {in }}^{\mathrm{A}} \mid n_{\Omega}\right\rangle_{\text {out }}^{\mathrm{B}}\left\langle\left. 0\right|_{\text {in }} ^{\mathrm{A}}\left\langle\left.(n+1)_{\Omega}\right|_{\text {out }} ^{\mathrm{B}}\right)\right],\right.
\end{aligned}
$$

where $T_{r}=\tanh r_{\Omega}$ and $C_{r}=\cosh r_{\Omega}$. The expression for $\rho_{A-\text { hor }}$ is obtained by exchanging $q_{R}$ and $q_{L}$ and 'out' by 'hor' in the equation above.

The entanglement monotone that we will compute, the negativity [20, is the sum of the negative eigenvalues of the partial transposed density matrix of the quantum state for which we want to evaluate its degree of distillable entanglement. To compute it we first take partial transposes in $\rho_{A-\text { out }}$ and $\rho_{A \text {-hor }}$ (which is the transpose only with respect to Alice's indices). This yields

$$
\begin{aligned}
& \rho_{A-\text { out }}^{T_{\mathrm{A}}}=\sum_{n=0} \frac{T_{r}^{2 n}}{2 C_{r}^{2}}|0\rangle_{\text {in }}^{\mathrm{A}}\left|n_{\Omega}\right\rangle_{\text {out }}^{\mathrm{B}}\left\langle0 | _ { \text { in } } ^ { \mathrm { A } } \left\langle\left. n_{\Omega}\right|_{\text {out }} ^{\mathrm{B}}\right.\right. \\
& +\sum_{n=0}(n+1) \frac{T_{r}^{2 n}}{2 C_{r}^{4}}\left(q _ { \mathrm { R } } ^ { 2 } | 1 \rangle _ { \text { in } } ^ { \mathrm { A } } | ( n + 1 ) _ { \Omega } \rangle _ { \text { out } } ^ { \mathrm { B } } \langle 1 | _ { \text { in } } ^ { \mathrm { A } } \langle ( n + 1 ) _ { \Omega } | _ { \text { out } } ^ { \mathrm { B } } + q _ { \mathrm { L } } ^ { 2 } | 1 \rangle _ { \text { in } } ^ { \mathrm { A } } | n _ { \Omega } \rangle _ { \text { out } } ^ { \mathrm { B } } \left\langle\left.1\right|_{\text {in }} ^{\mathrm{A}}\left\langle\left. n_{\Omega}\right|_{\text {out }} ^{\mathrm{B}}\right)\right.\right. \\
& +\sum_{n=0} \sqrt{(n+1)(n+2)} \frac{T_{r}^{2 n+1}}{2 C_{r}^{4}} q_{\mathrm{R}} q_{\mathrm{L}}\left(| 1 \rangle _ { \text { in } } ^ { \mathrm { A } } | n _ { \Omega } \rangle _ { \text { out } } ^ { \mathrm { B } } \langle 1 | _ { \text { in } } ^ { \mathrm { A } } \langle ( n + 2 ) _ { \Omega } | _ { \text { out } } ^ { \mathrm { B } } + | 1 \rangle _ { \text { in } } ^ { \mathrm { A } } | ( n + 2 ) _ { \Omega } \rangle _ { \text { out } } ^ { \mathrm { B } } \left\langle\left.1\right|_{\text {in }} ^{\mathrm{A}}\left\langle\left. n_{\Omega}\right|_{\text {out }} ^{\mathrm{B}}\right)\right.\right. \\
& +\sum_{n=0} \sqrt{n+1} \frac{T_{r}^{2 n+1}}{2 C_{r}^{3}}\left[q _ { \mathrm { R } } \left(| 1 \rangle _ { \text { in } } ^ { \mathrm { A } } | n _ { \Omega } \rangle _ { \text { out } } ^ { \mathrm { B } } \langle 0 | _ { \text { in } } ^ { \mathrm { A } } \langle ( n + 1 ) _ { \Omega } | _ { \text { out } } ^ { \mathrm { B } } + | 0 \rangle _ { \text { in } } ^ { \mathrm { A } } | ( n + 1 ) _ { \Omega } \rangle _ { \text { out } } ^ { \mathrm { B } } \left\langle\left.1\right|_{\text {in }} ^{\mathrm{A}}\left\langle\left. n_{\Omega}\right|_{\text {out }} ^{\mathrm{B}}\right)\right.\right.\right. \\
& +q_{\mathrm{L}}\left(|1\rangle_{\text {in }}^{\mathrm{A}}\left|(n+1)_{\Omega}\right\rangle_{\text {out }}^{\mathrm{B}}\left\langle\left. 0\right|_{\text {in }} ^{\mathrm{A}}\left\langle\left. n_{\Omega}\right|_{\text {out }} ^{\mathrm{B}}+\mid 0\right\rangle_{\text {in }}^{\mathrm{A}} \mid n_{\Omega}\right\rangle_{\text {out }}^{\mathrm{B}}\left\langle\left. 1\right|_{\text {in }} ^{\mathrm{A}}\left\langle\left.(n+1)_{\Omega}\right|_{\text {out }} ^{\mathrm{B}}\right)\right],\right.
\end{aligned}
$$

where, as above, the expression for $\rho_{A-\mathrm{A} o r}^{T_{\mathrm{A}}}$ is obtained by exchanging $q_{R}$ and $q_{L}$ and 'out' by 'hor'.

As it is the case of the accelerated-observer scenario, the diagonalisation of the infinite-dimensional partial transposed density matrices $\rho_{A-\text { hor }}^{T_{\mathrm{A}}}$ and $\rho_{A-\text { hor }}^{T_{\mathrm{A}}}$ can be carried out only numerically since, with the exception of the case $q_{\mathrm{R}}=1$, no block-diagonalisation can be performed.

Figure 2 shows the result of the calculations. The negativity 'A-out' as a function of the mass of the forming black hole and the frequency of the probed 'out' mode is shown as solid blue lines. The negativity 'A-hor' is plotted in the same figure as dashed red lines.

We see that varying the parameter $q_{\mathrm{R}}$ we are basically controlling whether Alice's state will have more quantum correlations with the modes that will reach the asymptotic future or the modes that will fall into the horizon. This is best seen in the large black hole mass limit, where there is an exact trade-off between the correlations that Alice's mode has with infalling and outgoing modes.

We can also see that for very small black holes, all entanglement is completely degraded: when $m \rightarrow 0$ no entanglement survives either with the modes that fall into the horizon or the modes that will reach the future. As the mass of the black hole (or the frequency of the probed mode) is increased, the correlations quickly become insensitive to the presence of the horizon, as one can expect taking a look at the quantum effects induced by gravity in the presence of a black hole: They become stronger as the mass of the black hole is closer to zero. This suggests that this can be understood in a pictorial way as a limit in which the Hawking-like radiation spoils all correlations contained in the state.

As it is well known [21, if Bob can only measure modes in the asymptotic future, he will see the vacuum state $|0\rangle_{\text {in }}$ as a thermal state. Indeed, if we compute how the 'in' vacuum is seen by observers in the asymptotic future we obtain 


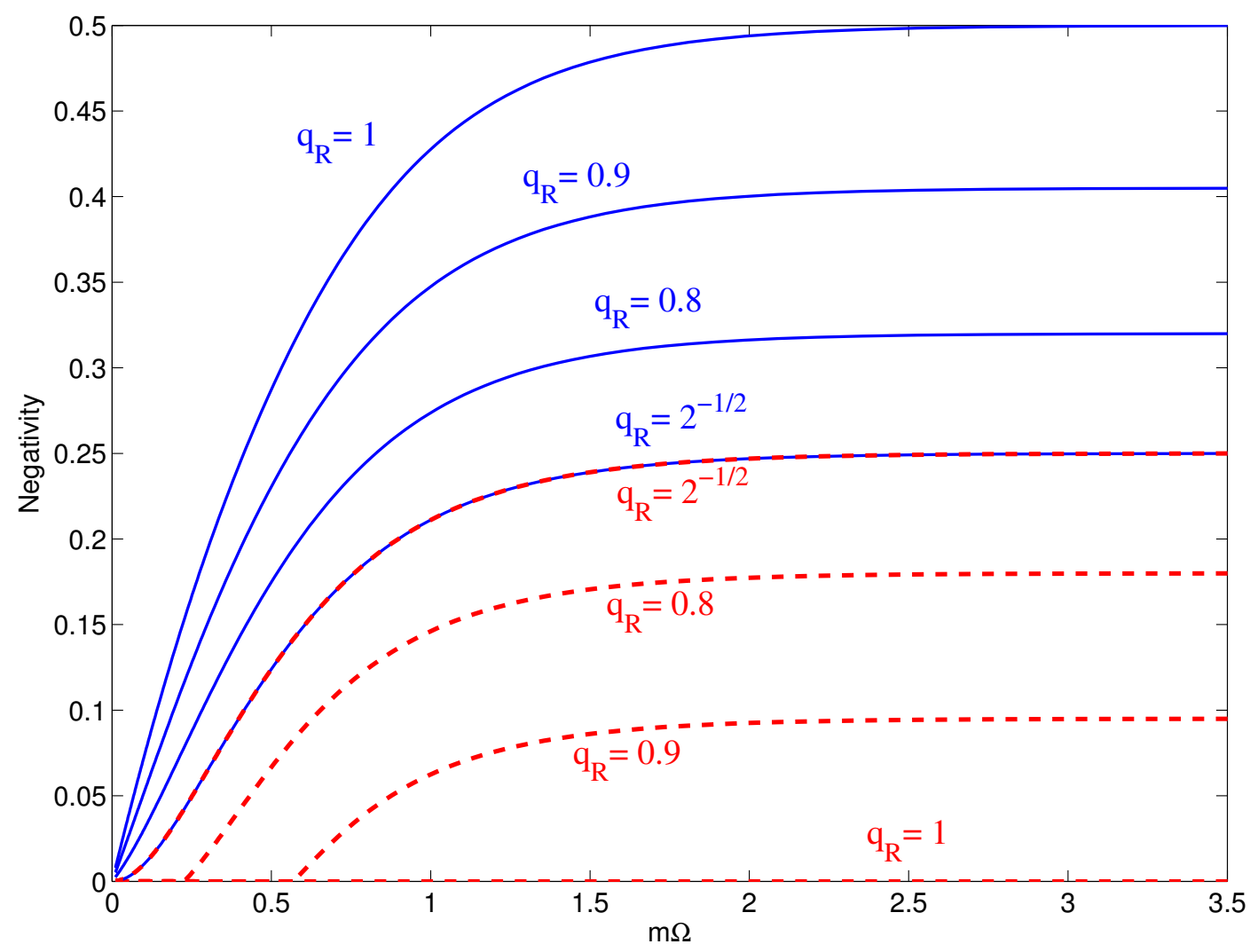

Figure 2. Negativity of $\rho_{A-\text { out }}$ (solid blue line) and $\rho_{A-\text { hor }}$ (red dashed line) as a function of the product of mass of the black hole and probed frequency for various choices of the past modes 15 . Note that no entanglement survives the singular black hole limit $(m \rightarrow 0)$ where the Hawking temperature is divergent.

that $\rho_{\text {out }}^{|0\rangle_{\text {in }}}=\operatorname{tr}_{\text {hor }}\left(|0\rangle_{\text {in }}\langle 0|\right)=\prod_{\omega} \rho_{\text {out }, \omega}$, where

$$
\rho_{\text {out }, \omega}=\frac{1}{\left(\cosh r_{\omega}\right)^{2}} \sum_{n=0}^{\infty}\left(\tanh r_{\omega}\right)^{2 n}\left|n_{\omega}\right\rangle_{\text {out }}\left\langle n_{\omega}\right|
$$

This is a thermal radiation state whose temperature is $T_{\mathrm{H}}=(8 \pi m)^{-1}$. So if that happens with the vacuum state it would be a reasonable hand-waving argument that, if instead of the vacuum, we consider a pre-existing non-trivial entangled state such as that of equation (16), the thermal-like noise could impair the ability of the observers of acknowledging quantum correlations in the system.

However one has to be very careful when thinking to what extent this behaviour can be naively associated to the Hawking thermal noise. To begin with, we are not considering the vacuum state, but rather an entangled state of field excitations. The process of change of basis and tracing out of the modes that fall into the event horizon is not as trivial as for the vacuum case. In fact, it has been shown that in the Rindler scenario 22 and beyond the so-called single mode approximation with some choices of the state, the accessible entanglement for an accelerated observer may behave in a non-monotonic way, as opposed to the first results reported in [2, 3, 7]. This is due to inaccessible correlations in the initial states becoming accessible to the accelerated observer when his proper Fock basis changes as acceleration varies. While this phenomenon was highlighted in [22] for the Rindler case, for an analogous choice of the modes [15], a similar behaviour would be expected in the dynamic scenario analysed here.

Let us conclude with a note of warning: The modes analysed here as tools to study entanglement behaviour in gravitational collapse have very nice properties but due to their highly non-monochromatic nature and non-localisation they are modes that can arguably be difficult to prepare and measure in an hypothetical experiment. This said, this tool will allow us to simplify the calculations so that we can extract fundamental results in settings in which other 
techniques have proven not operational, much in the same fashion as the introduction of Unruh modes [7. has allowed progress in our understanding of quantum correlations from non-inertial perspectives. One has to keep in mind that the modes used here share some fundamental properties with the standard monochromatic 'in' modes, and that they form a complete basis of solutions to the field equations. This means that any physically conceivable state can be expressed as a superposition of modes as the ones studied here.

\section{Conclusion and Future research}

We have analysed the behaviour of quantum entanglement present in some initial field state when a gravitational collapse occurs in the background. The quantum correlations in that state are perturbed by the formation of the event horizon, mixing the quantum state originally prepared, and therefore, degrading the original correlations. We have done so by adapting the tools developed in the analysis of entanglement in the context of the Unruh-Hawking effect [2, 11] to go beyond what was known as 'single mode approximation' 7].

We have shown, that similarly to the infinite acceleration limit in accelerated scenarios [2, 6, 7], and similar to the stationary eternal blackhole scenario [11, entanglement is completely degraded when we consider singular black holes $(m \rightarrow 0)$ for which the Hawking temperature diverges.

A trivial extension of the results obtained here is considering that the appearance of an event horizon affects the ability to access the full state for both Alice and Bob. In these cases, and for maximally entangled states of a scalar field, entanglement will be arguably degraded more quickly than in the case where only Bob is affected by the collapse, much in a similar way as it happens in the acceleration scenario [23. Extending this result to the case where both observers measure after the horizon is created is somewhat straightforward with the tools developed here, being mainly a matter of a more complicated calculation, whereas the results are arguably going to be qualitatively the same.

The next natural step is to introduce localised measurements that will endow the entanglement degradation phenomena reported here with operational meaning. Using for example, localised projective measurements as in [19] more physical scenarios can be analysed.

\section{Acknowledgements}

We would like to thank Robert B. Mann and Tim Ralph for their kind invitation to contribute to the Focused issue of Class. and Quantum Grav. on Relativistic Quantum Information. This work was supported by the Spanish MICINN/MINECO Projects FIS2011-29287, FIS2008-06078-C03-03, FIS2011-30145-C03-02, the CAM research consortium QUITEMAD S2009/ESP-1594, and the Consolider-Ingenio 2010 Program CPAN (CSD2007-00042).

\section{References}

[1] P. M. Alsing and G. J. Milburn, "Teleportation with a uniformly accelerated partner," Phys. Rev. Lett. 91, 180404 (2003).

[2] I. Fuentes-Schuller and R. B. Mann, "Alice falls into a black hole: Entanglement in non-inertial frames," Phys. Rev. Lett. 95, 120404 (2005).

[3] P. M. Alsing, I. Fuentes-Schuller, R. B. Mann, and T. E. Tessier, "Entanglement of Dirac fields in noninertial frames," Phys. Rev. A 74, 032326 (2006).

[4] J. León and E. Martín-Martínez, "Spin and occupation number entanglement of Dirac fields for noninertial observers," Phys. Rev. A 80, $012314(2009)$.

[5] E. Martín-Martínez and J. León, "Fermionic entanglement that survives a black hole," Phys. Rev. A 80, 042318 (2009).

[6] E. Martín-Martínez and J. León, "Quantum correlations through event horizons: Fermionic versus bosonic entanglement," Phys. Rev. A 81, 032320 (2010).

[7] D. E. Bruschi, J. Louko, E. Martín-Martínez, A. Dragan, and I. Fuentes, "Unruh effect in quantum information beyond the single-mode approximation," Phys. Rev. A 82, 042332 (2010).

[8] E. M.-M. N. Friis, P. Köhler and R. A. Bertlmann., "Residual entanglement of accelerated fermions is not nonlocal," Phys. Rev. A 84, $062111((2011))$.

[9] D. E. Bruschi, I. Fuentes, and J. Louko, "Voyage to Alpha Centauri: Entanglement degradation of cavity modes due to motion," Phys. Rev. D 85, 061701 (2012).

[10] N. Friis, D. E. Bruschi, J. Louko, and I. Fuentes, "Motion generates entanglement," Phys. Rev. D 85, 081701 (2012).

[11] E. Martín-Martínez, L. J. Garay, and J. León, "Unveiling quantum entanglement degradation near a Schwarzschild black hole," Phys. Rev. D 82, 064006 (2010).

[12] J. L. Ball, I. Fuentes-Schuller, and F. P. Schuller, "Entanglement in an expanding spacetime," Phys. Lett. A 359, 550 (2006).

[13] E. Martín-Martínez, L. J. Garay, and J. León, "Quantum entanglement produced in the formation of a black hole," Phys. Rev. D 82, 064028 (2010).

[14] R. Balbinot, A. Fabbri, S. Fagnocchi, A. Recati, and I. Carusotto, "Nonlocal density correlations as a signature of Hawking radiation from acoustic black holes," Phys. Rev. A 78, 021603 (2008).

[15] A. Fabbri and J. Navarro-Salas, Modeling black hole evaporation (World Scientific, London (United Kingdom), 2005).

[16] R. Schützhold and W. G. Unruh, "Quantum correlations across the black hole horizon," Phys. Rev. D 81, 124033 (2010).

[17] B. Horstmann, B. Reznik, S. Fagnocchi, and J. I. Cirac, "Hawking Radiation from an Acoustic Black Hole on an Ion Ring," Phys. Rev. Lett. 104, 250403 (2010).

[18] P. M. Alsing, D. McMahon, and G. J. Milburn, "Teleportation in a non-inertial frame," J. Opt. B: Quantum Semiclass. Opt. 6, S834 (2004). 
[19] A. Dragan, J. Doukas, E. Martin-Martinez, and D. E. Bruschi, "Localised projective measurement of a relativistic quantum field in non-inertial frames," arXiv:1203.0655 .

[20] G. Vidal and R. F. Werner, "A computable measure of entanglement," Phys. Rev. A 65, 032314 (2002).

[21] S. W. Hawking, "Black hole explosions?," Nature 248, 30 (1974).

[22] M. Montero and E. Martín-Martínez, "The entangling side of the Unruh-Hawking effect," JHEP 2011, 1 (2011).

[23] G. Adesso, I. Fuentes-Schuller, and M. Ericsson, "Continuous-variable entanglement sharing in non-inertial frames," Phys. Rev. A 76, $062112(2007)$. 